\title{
Correction to: Application of Plant-Based Natural Product to Synthesize Nanomaterial
}

Mohammad Khajeh Mehrizi and Zahra Shahi

\section{Correction to:}

Chapter 3 in: M. Srivastava et al. (eds.),

Nanomaterials in Biofuels Research,

Clean Energy Production Technologies,

https://doi.org/10.1007/978-981-13-9333-4_3

The book was inadvertently published without including the name of the second author (Zahra Shahi) in Chapter 3. This has now been corrected. 